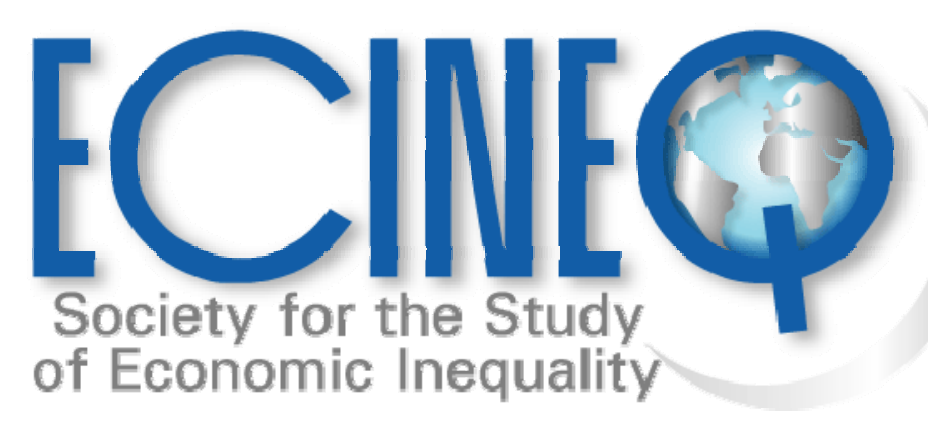

Working Paper Series

Comparing Multidimensional Indices of Inequality: Methods and Application

María Ana Lugo

ECINEQ WP 2005 - 14 


\title{
Comparing Multidimensional Indices of Inequality: Methods and Application*
}

\author{
María Ana Lugo ${ }^{\dagger}$ \\ Department of Economics, University of Oxford
}

This version: November 2005

\begin{abstract}
The paper provides an examination into the measures of multidimensional inequality proposed in the past few years, their properties and majorization criteria. It offers a generalisation of Bourguignon index proposed so that it includes Tsui measures (1999) while preserving the virtues of Maasoumi's method (1986) of explicitly acknowledging the role of parameters relevant of multivariate settings. An application to the Argentine data is provided to illustrate the decisions involved in the process of applying these measures and the usefulness of having appropriate criteria for the choice.
\end{abstract}

Keywords: Inequalities, Multidimensional Distributions, Well-being, Multivariate indices.

JEL Classification: D30, D31, D63, I10, I21, I31, I32, O15, O54

\footnotetext{
* The paper was presented at the First Meeting of the Society for the Study of Economic Inequality in Mallorca and QEH 50 th Anniversary Conference. I am most grateful for the supervision at different stages of Prof Sudhir Anand, Prof Stefan Dercon, and Rosemary Thorp. I also thank Gordon Anderson, Esfandiar Maasoumi, Ernesto Savaglio and Jacques Silver for their excellent comments; Koen Decancq for insightful email discussions, parts of which are reflected in the paper; and participants of the ECINEQ $1^{\text {st }}$ Meeting, Palma de Mallorca, July 2005. All errors are mine.

†Contact details: maria.lugo@economics.ox.ac.uk. Dept Economics, University of Oxford, Manor Road Building, Manor Road, OX1 3UQ, Oxford, UK.
} 


\section{Introduction}

In the area of income distribution measurement, specialists have designed a great number of analytical tools (measures and methods) to compare distributions, some of them widely accepted and much studied. Extending the traditional definition of well-being to account for multiple dimensions requires extending also the existing techniques or, if necessary, designing new ones. This adaptation is not always straightforward and in the process certain value judgements are unavoidable.

The formal analysis of multidimensional inequality was pioneered by Kolm (1977). One of his main contributions was to provide a number of multivariate generalisations of the Pigou-Dalton principle of transfer. Atkinson and Bourguignon (1982) followed Kolm's perspective by developing dominance criteria to determine the conditions under which one multivariate distribution was more unequal than another. These dominance principles take account of the correlation between the distributions of the different components of well-being bringing a new and distinctive element of the multidimensional analysis.

In practice, it is highly unlikely that dominance conditions in the multivariate context will be satisfied, hence leading to incomplete rankings of distributions. In these cases, multidimensional inequality indices become particularly attractive. They synthesise the information of each multidimensional distribution into a real-value measurement. Similarly to the univariate approach, multidimensional inequality measures have been derived using both an axiomatic approach (see Tsui 1995; 1999) or a direct 'ad hoc' one (Maasoumi 1986).

The present paper provides an examination of some of the measures of multidimensional inequality proposed in the past years. The aim is to assess them critically whilst comparing the properties satisfied (distributional and otherwise). It will also emphasise the distinct roles of the parameters included in the formulae, not always made explicit by the authors. It is believed here that disregarding them renders the interpretation and proper understanding of the measures much more complex. We present here a generalisation of an index proposed by Bourguignon (1999) capturing the advantages of both Maasoumi and Tsui indices studied. The paper extends the analysis of a previous work (Lugo 2004) both on the measures examined and depth of the assessment, and on the application to Argentina utilising individual level data instead of aggregate (provincial) level data. 
The paper is structured as follows: in the first section, the properties and majorization criteria are presented. Complete additive separability - desirable property in the univariate context - cannot be sustained together with a majorization criterion related to the role of correlation between dimensions in the measure. The second section presents three sets of multidimensional inequality measures proposed in the literature (Bourguignon 1999; Maasoumi 1999; Tsui 1999), compares their characteristics and extends the family of Bourguignon indices to include Tsui's index. The third section provides an application to the Argentine data to illustrate the decisions to be made in the process of applying these measures and the usefulness of having appropriate criteria for the choice. Section four concludes.

\section{Definitions}

Let $k=[1,2, \ldots K]$ be the set of attributes, $i=[1,2, \ldots n]$ the set of individuals (households, provinces, etc. ) with $n>1$, and $\mathrm{M}(n)$ the set of $n \times K$ matrices with non-negative elements. A multidimensional distribution is an $n \times K$ matrix $X=\left(x_{i k}\right) \in \mathrm{M}(n)$, where $x_{i k} \in \mathbb{R}_{+}$. Let $\mathbf{x}_{\mathbf{i}}$ denote the row vector of attributes for the $i$-th individual and $\mathbf{x}^{\mathrm{k}}$ the (column) vector of the $k$-th attribute among individuals, in other words, the marginal distribution of each dimension.

A multidimensional index of inequality is a function $I^{n}(X): \mathfrak{M}(n) \mapsto \Re$, i.e. a continuous realvalued function summarising the information about a given distribution. Inevitably, each $I$ implicitly or explicitly satisfies a series of properties together with some majorization criteria. In the literature, there are two distinct identified procedures: two-stage approach and one-step multidimensional inequality indices. In the former (Bourguignon 1999; Maasoumi 1986; 1999), the aggregation is done explicitly, arriving first at a single composite measure for each individual (household) expressed in some 'well-being unit'; applying, secondly, some univariate inequality index. The latter (Tsui 1995; 1999), on the other hand, have implicit in the inequality measure the valuation function of attributes.

In both strategies decisions are made inevitably over (1) the weighting structure $\left(w_{1}, w_{2}, \ldots, w_{K}\right)$, that is, the extent to which each dimension is assumed to contribute to individuals' well-being; (2) the degree of substitution between each pair of attributes $\left(\beta_{\mathrm{km}}\right.$, for all $\left.k, m: 1,2, \ldots K\right)$; and (3) the degree of inequality aversion in the well-being space $(\alpha)$. The value of these parameters will normally depend on a set of desirable properties of the inequality index and the underlying aggregation function ${ }^{2}$.

\footnotetext{
2 Weighting is usually done in one of these ways: (a) treating all dimensions equally - equal weighting $\left(w_{1}=w_{2}\right.$
} 
This is consistent both with a welfarist approach in which the function represents the way the society values individual levels of attributes (social welfare function, SWF) -i.e. through the intermediary of utility - and any non-welfarist line which it views the composite index merely as one possible form of summarising the information over individuals' attributes (social evaluation function, SEF) - individuals' well-being depending directly on their attributes (Tsui 1999).

\subsection{Properties}

The following set of properties - straightforward generalisations from the univariate context as introduced by Shorrocks (1984) - is often defined as the non-distributional properties for multidimensional measures of inequality (see Tsui 1999) ${ }^{3}$, set as properties or axioms.

Continuity $(\mathbf{C N}): I^{n}(X)$ is continuous, that is, a small change in any attribute does not result in abrupt changes in the measurement of inequality.

Anonymity (AN) - wrt individuals: $I^{n}(X)$ does not depend on the identity of individuals, i.e. $I^{n}(X)=I^{n}(P X)$ where $P$ is an $n \times n$ permutation matrix which interchanges the coordinates of an $n$-vector $\mathbf{x}_{\mathrm{i}}$ so that $P \mathbf{x}$ is a permutation of $\mathbf{x}$.

Normalisation (NM): If every individual has the same bundle of attributes $\left(\mathbf{x}_{\mathbf{i}}=\mathbf{x}^{*}\right)$ then there is complete equality and the degree of inequality is normalised to zero, i.e. $I^{n}\left(\mathbf{x}^{*}, \mathbf{x}^{*}, \ldots, \mathbf{x}^{*}\right)=0$.

Replication Invariance (RI): replicating $r$ times the population without changing the distribution of attributes does not change the value of $I^{n}(X)$, i.e. $I^{n}(X, X, \ldots, X)=I^{n}(X)$ for any $r \in \mathbb{N}$

Scale Invariance (SI). An equal proportional increase of all $K$ attributes to all $n$ individuals does not affect the measurement of multidimensional inequality. If each $k$ attribute is multiplied by the

$=\ldots=w_{K}$ ), 'from an 'agnostic' attitude or from the lack of information about some kind of 'consensus' view" Brandolini and D'Alessio (2001), 22. - ; (b) setting weights according to some normative criterion in which at least one $w_{i} \neq w_{j}$; (c) using data-driven techniques, i.e. multivariate procedures such as factor analysis, principal component or cluster analysis to derive weights. If market prices for all attributes were available, we can also use them as the basis for the weights. Clearly, for many of the dimensions included in any concept of well-being, market prices do not exist. Irrespectively of the criteria used, we could also follow Sen's recommendation to utilise a range of weights rather than a single set for robust results (Sen, et al. (1987), ).

${ }^{3}$ On the reasonableness of desirable properties for inequality measures, Tsui's comment becomes relevant. An experiment on university students carried out by Ameil and Cowell (1992) show that "as expected there is no consensus on the acceptability of these axioms. In the final analysis, inequality involves interpersonal comparison. The axioms are in most cases ethical in nature. In the multidimensional context, there is likely to be even less consensus on the properties of multidimensional indices" (Tsui (1995), 254., the underlying is mine). Also, 'sensible properties' require that they are so "relative to 'usage', and/or logical practical (mathematical) considerations" (Maasoumi (1993), 167.) 
constant $\lambda$ for all individuals, $I^{n}(X)$ will not change, that is, it is homogeneous of degree zero with respect to each column of $X$, i.e. $I^{n}(X D)=I^{n}(X)$, where $D=\operatorname{diag}(\lambda, \lambda, \ldots, \lambda), \lambda>0, i=1,2, \ldots K$. A measure satisfying this condition is said to be a relative inequality measure, i.e. what matters is the relative positions on individuals in the multivariate distribution, thus it is not affected by changes in the units of measurement ${ }^{4}$.

Translation Invariance (TI): adding a constant $a$ to each attribute to all individuals does not change the level of inequality, i.e. $I^{n}(X+a 1)=I^{n}(X)$, where 1 is a distribution matrix whose entries are all equal to one. In other words, $I$ (.) should be invariant to a common change in the origins from which the quantities of the attributes are measured. This property is relevant for absolute measures of inequality 5 .

Decomposability. In the weakest version, it implies that overall inequality can be expressed as a general function of the subgroup means, population sizes, and inequality values.

$\left.I^{n}\left(X^{1}, X^{2}\right)=A\left[I^{n 1}\left(X^{1}\right), \mu_{1}, n_{1}, I^{n 2}\left(X^{2}\right), \mu_{2}, n_{2}\right)\right]$, where $X=\left(X^{1}, X^{2}\right)$ e $\mathrm{M}(n), X^{1}$ is a submatrix of $\mathrm{X}$ of $n_{1}$ rows/columns (individuals/attributes' vectors) with $\mu_{1}$ as their vector of means, and similarly for $X^{2}, n_{2}$, and $\mu_{2}$. The aggregative function $A$ is continuous and strictly increasing in $I^{n 1}$ $\left(X^{1}\right)$ and $I^{n^{2}}\left(X^{2}\right)$. We distinguish decomposability by population subgroups (DP) and by attributes (DA).

Additive Separability (AS) is a stricter version of the D property. It implies that individual components of welfare are judged without reference of other persons' welfare. A measure is said to be additive separable when it can be decomposed in such a way that "The total inequality can be expressed as the sum of a 'within group' inequality term and a 'between-group' term, where the within-group contribution is itself a weighted sum of the sub-group inequality values" (Shorrocks 1980, 614)

\footnotetext{
${ }^{4}$ Strong Scale Invariance (SSI) Tsui (1995) proposes this stronger version of (SI). If each $k$ attribute is multiplied by a constant $\lambda_{k}$ for all individuals, $I^{n}(X)$ will not change, that is, it is homogeneous of degree zero with respect to each column of $X$, i.e. $I^{\prime \prime}(X D)=I^{\prime \prime}(X)$, where $D=\operatorname{diag}\left(\lambda_{1}, \lambda_{2}, \ldots, \lambda_{K}\right), \lambda_{k}>0, \mathrm{i}=1,2, \ldots$ $K$. This property merely says that the measure should be relative in each of the k-th dimensions. Bourguignon (1999) has argued against this property, as it is reasonable to believe that if, for instance, incomes are doubled the contribution of inequalities in health status to overall multidimensional inequality may be expected to be quite different. "As a matter of fact, not to exhibit the scale invariance property which is commonly postulated in the unidimensional case, may be an important and interesting feature of multidimensional inequality measures" (Bourguignon (1999), 479.). Scale invariance and Strong Scale Invariance inequality measure properties are equivalent to requiring the underlying social evaluation relation to be homothetic and strong homothetic (see Weymark (2006), ).

${ }^{5}$ Strong Translation Invariance (STI): adding a constant $a_{k}$ to each $k$ attribute to all individuals does not change the level of inequality, i.e. $I^{n}(X+A)=I^{\prime \prime}(X)$, where $A$ is a $n \times K$ matrix with identical rows $\left(a_{1}, a_{2}\right.$, $\left.\ldots, a_{k}\right)$; i.e. $I($.$) is invariant to independent changes in origins. This is a generalisation of the 'leftist' notion$ of inequality according to Kolm's formalisation Tsui (1995), . Translation invariance and Strong Translation Invariance inequality index properties are equivalent to requiring the underlying social evaluation relation to be translatable and strongly translatable (see Weymark (2006), ). SSI and STI would not be appropriate if attributes are measured in the same units.
} 
$I(X)=I\left(X_{1}, \ldots, X_{G}\right)=\sum_{g}^{G} w_{g} I\left(X_{g}\right)+B$, where $X_{1} \ldots X_{G}$ represents partition of the distribution of $\mathbf{X}$ into $\mathrm{G}$ groups and B represents the between-group component. Once again, we can distinguish additive separability by population subgroups (ASP) and by attributes (ASA).

These properties are interesting both because they permit computing overall inequality from subgroup data and facilitate the study the sources of inequality. In principle, they will be particularly useful when working with limited datasets. Frequently, only aggregated data is available; i.e. for each aggregated unit (such as regions, states, provinces) we might have average levels and inequality value for each attribute, most commonly from different sources. Thus, it would seem particularly useful to be able to work with inequality indices which allow us to work in such an environment. If one is able to obtain some estimates of correlations between dimensions from other sources DA will be enough; a stricter (ASA) is not required.

\subsection{Majorization criteria}

The list of properties satisfied by $I^{n}(X)$ should be completed with majorization or dominance criteria, that is to say, with "partial orders ranking distributions of attributes by their degree of inequality" (Tsui 1999, 146) or distributional properties. The study of majorization criteria in the context of multidimensional indices of inequality was pioneered by Kolm (1977). The first two majorization criteria below deal with the spread of the distribution of attributes (extensions of the univariate Pigou-Dalton principle); the third addresses the issue of correlation between attributes' distributions (specific of the multidimensional distribution context).

One dimensional Pigou-Dalton condition extends to two distinct dominance criteria in a multiple dimensional context, which are not always equivalent.

Uniform Pigou-Dalton Majorization (UPD) ${ }^{6} .(X, X T) \in \mathrm{UPD}$ where $T$ is a finite product of Pigou-Dalton matrices and it is not a permutation matrix. A Pigou-Dalton matrix $T$ is one such that $T=\lambda E+(1-\lambda) Q, 0<\lambda<1$, where $E$ is an identity matrix and $Q$ is a permutation matrix which transforms other matrices interchanging two rows. $(X, X T) \in \mathrm{UPD} \Rightarrow I^{n}(X)>$ $I^{n}(X T)$.

${ }^{6}$ Also known as 'chain majorization' in Marshall and Olkin (1979), . 
Uniform Majorization (UM) ${ }^{7}$. $(X, X B) \in \mathrm{UM}$ where $B$ is a $(n \times n)$ bistochastic matrix and $Y$ cannot be derived by permuting by the columns of $X$. Intuitively, multiplying $X$ by a bistochastic matrix renders the distributions of attributes less spread out, so that $(X, X B) \in \mathrm{UM} \Rightarrow I^{n}(X)$ $>I^{n}(X B)$.

Both UM and UPD measure the spread of the attributes. The difference rest in the fact that UPD implies the property to hold for each of the marginal distribution of attributes, but the converse does not always hold unless $K=1$ or $n=2$. The reason is that any $T$ matrix is a nonpermutation bistochastic matrix but there exist some $B$ matrix that cannot be expressed as product of Pigou-Dalton matrices. In other words, if each univariate distribution of dimension in $X$ is Pigou-Dalton majorized by $X T$, still it does not necessarily imply that the multidimensional matrix X is Pigou-Dalton majorized by XT (Marshall and Olkin 1979). UPD implies UM but the reverse does not need to hold

Kolm shows that UM is satisfied under one of the following (from Theorem 5 and 6, Kolm 1977): Schur-convex inequality measures; Schur-concave SEF; individualistic and concave individual aggregator function and Schur-concave SEF; individualistic and concave individual aggregator function and quasi-concave SWF; individualistic and concave individual aggregator function and additive separable SEF8.

Tsui (1999) introduces a majorization criterion capturing a key concept in Atkinson-Bourguignon (1982)'s paper related to the correlation among attributes when marginal distributions are unchanged, which was not previously needed. This is, in effect, the 'innovation' of multivariate inequality axiomatisation, and it is what renders the multidimensional case "intrinsically different from the one-dimensional case and makes assumptions on the sign of [cross-derivatives of the

\footnotetext{
7 a.k.a. 'Majorization' in (1979), ; 'Multidimensional version of the Principle of Transfer' in Maasoumi (1986), , Maasoumi (1999), ; 'Multidimensional generalization of the Pigou-Dalton principle' in Tsui (1995), ; and Uniform Majorization in Weymark (2006), . In a welfarist context, UM is equivalent to Lorenz dominance when the utility function belongs to the family of functions that are continuous and strictly concave (Kolm (1977), , Tsui (1999), ).

${ }^{8}$ Definitions: Schur-convex and Schur-concave (or S-concave) as defined by Marshall and Olkin (1979):

A real-valued function $\Phi$ defined on $\mathcal{A} \subset \mathbb{R}$ is said to be Schur-convex on $\mathcal{A}$ if $\mathbf{x} \succ \mathbf{y}$ on $\mathcal{A}$ $\Rightarrow \Phi(\mathbf{x}) \geq \Phi(\mathbf{y})$ and Schur-concave on $\mathcal{A}$ if $\mathbf{x} \succ \mathbf{y}$ on $\mathcal{A} \Rightarrow \Phi(\mathbf{x}) \leq \Phi(\mathbf{y})$. Also, if the $\Phi(\mathrm{x})$ is Schur-convex (concave), the function $-\Phi(\mathrm{x})$ is Schur-concave (convex) and any monotonic increasing transformation of a Schur-concave (convex) function is also Schur-concave (convex). See Boche and Jorswieck (2004), $3 .$. In the unidimensional context, S-concavity is a weaker requirement, given symmetry, than strict concavity and strict quasi-concavity; "it is equivalent to the Pigou-Dalton transfer condition, with symmetry" (Sen and Foster (1997), 134.)

We should be careful about notation as in this context $\mathbf{x} \succ \mathbf{y}$ on $\mathcal{A}, \mathbf{x}$ is majorized by $\mathbf{y}$, means in fact that $\mathbf{y}$ is "less spread out" than $\mathbf{x}$, that is, $\mathbf{y}$ is more equally distributed. It is standard to prefer $\mathbf{y}$ in terms of welfare if one values negatively inequality, but notation as coming from the 'Theory of Majorization' interprets $\succ$ in the explained form; Marshall and Olkin (1979), .
} 
underlying function] necessary" (Atkinson and Bourguignon 1982, 191)

Boland and Proschan (1988) define a correlation increasing transfer as one that redistributes the amount of attributes between individuals $i$ and $j$ in such a way that $i$ gets the lower of the two quantities of each attribute while $j$ gets the higher, while the distribution of attributes among everyone else remains constant. Thus, when two distributions $X, Y \in \mathfrak{M}(n)$ have equivalent marginal distributions in the $K$ dimensions but differ in a way such $Y$ is derived from $X$ by a correlation increasing transfer, UPD and UM cannot rank them. Formally, $Y$ may be derived from $X$ by a correlation increasing transfer if, for some rows $i$ and $j, i<$ $j, \mathbf{y}_{i}=\mathbf{x}_{i} \wedge \mathbf{x}_{j}$ and $\mathbf{y}_{j}=\mathbf{x}_{i} \vee \mathbf{x}_{j}, \mathbf{y}_{m}=\mathbf{x}_{m}, \quad m \notin\{i, j\}, \quad$ where $\mathbf{x} \wedge \mathbf{y}=\left(\min \left\{x_{1}, x_{2}\right\}, \ldots, \min \left\{x_{k}, y_{k}\right\}\right)$ and $\mathbf{x} \vee \mathbf{y}=\left(\max \left\{x_{1}, x_{2}\right\}, \ldots, \max \left\{x_{k}, y_{k}\right\}\right)$ (Boland and Proschan 1988).

Correlation-Increasing Majorization (CIM). $(X, Y) \in \mathrm{CIM} \Leftrightarrow Y$ can be derived from $X$ by permutation of rows and a finite number of correlation increasing transfers of attributes among individuals. In other words, more correlation between attributes, for given marginal distributions, should lead to less equal allocation, that is, $I^{n}(X) \leq I^{n}(Y)$. This majorization criterion captures the idea of compensating inequalities among different attributes 9 .

CIM majorization corresponds to the case where the social welfare function pertains to the class of functions $\mathfrak{A}^{-}$, i.e. the first derivatives with respect to each attribute is non-negative, and the cross-derivative is non-positive, and marginal distributions are identical. The sign of the crossderivative of the inequality index should be non-negative.

Bourguignon and Chakravarty criticise the use of CIM as a valid majorization criterion as it is implicitly assuming that attributes are substitutes, while "there is no a priori reason for a person to regard attributes as substitutes only. Some of the attributes can as well be complements" (Bourguignon and Chakravarty 2003, 36).

Note that, by definition, additive separability by attributes (ASA) will necessarily ignore the correlation between dimensions, hence ignoring one of the main elements of the multidimensional framework. In the next section, we will see how CIM and ASA are inconsistent. Given the data limitations, especially in developing countries, it might be worth

\footnotetext{
${ }^{9}$ Within the welfarist approach - hence the existence of utility functions intermediating attributes to wellbeing - CIM is included in the two Lorenz dominance majorizations; in the Weakly Utility-based Majorization (WUBM) for utility functions $u($.$) that are increasing and L-superadditive, and included in$ 'Utility-based Majorization' (UBM) for continuous and strictly concave $u($.$) s Tsui (1999), .$
} 
further investigating the possibility of adapting ASA in such a way as to exploit the data at hand. On the other hand, ASA will still be relevant in the analysis on intertemporal allocation when additivity $\left(U_{12}=0\right)$ is generally assumed, as pointed out in Atkinson and Bourguignon (1982).

\section{Multidimensional measures of inequality}

\subsection{Maasoumi $(1986,1999)$}

In a series of papers, Maasoumi proposes the use of a measure to compare multidimensional distributions of well-being, based on the theory of information (Hirschberg et al. 1991; Maasoumi 1986; 1999; Maasoumi and Nickelsburg 1988). The index is constructed in two steps, first aggregating attributes for each individual (obtaining some $S_{i}$ function of the $X_{i k}$ ) then applying a measure of inequality as in the univariate case. In both these steps, the author makes his choices based on "information theory", arguing its benefits as compared to an axiomatic approach ${ }^{10}$, hence choosing General entropy measures for both stages.

Based on the idea that different indicators of economic welfare are distributed differently, Maasoumi suggests an aggregator vector $S$ with a distribution that most closely represents the distributional information in each attribute - which he implicitly assumes to be a desirable property. In particular, he proposes a multivariate generalisation of the $\boldsymbol{G} \boldsymbol{E}$ measure of divergence (Kullback-Leibler distance) or closeness between the $K$ densities [as weighted sum of the pairwise 'divergence' terms] and arrives at a distance measure $D$ of the following form:

$$
D_{\beta}(S, X ; w)=\sum_{k=1}^{K} d_{k}\left\{\sum_{i=1}^{n} S_{i}\left[\left(\frac{S_{i}}{x_{i k}}\right)^{-\beta}-1\right] / \beta(\beta-1)\right\}
$$

The distribution of $S=\left(S_{1}, S_{2}, \ldots, S_{N}\right)$ which minimises $D_{\beta}$ produces the 'optimal' aggregation functions:

$$
S_{i} \propto \begin{cases}\left(\sum_{k=1}^{K} w_{k} X_{i k}^{\beta}\right)^{1 / \beta} & , \beta \neq 0 \\ \prod_{k}^{K} x_{i k}^{w_{k}} & , \beta=0\end{cases}
$$

Where $w_{k}=d_{k} / \sum_{j=1}^{K} d_{j}$ is the weight given to the $k$-th attribute in the total aggregator function.

\footnotetext{
${ }^{10}$ Maasoumi argues that as the GE divergence is itself justified by a set of desirable axioms of information theory similar to those posed by the followers of an axiomatic approach, it cannot be blamed to ignore them altogether. This was already made cleared by Shorrocks when first using an axiomatic approach to derive inequality measures. Shorrocks (1980),
} 
It is straightforward to see that $S_{i}$ is the general weighted mean with the familiar formulation of the CES and Cobb-Douglas functions as special cases $^{11}$. Maasoumi interprets this aggregator function as the well-being indicator for person $i$. For strictly positive $x_{i k}, S_{i}$ is defined for all values of $\beta$.

The parameter $\beta$ is related to the degree of substitutability between attributes and determines the shape of the contours for all pair of attributes - identical for all pairs. The elasticity of substitution is given by $\sigma=\frac{1}{1-\beta}$. The smaller the $\beta$, the smaller is the elasticity of substitution between attributes. In the limit, as $\beta \rightarrow-\infty, \sigma \rightarrow 0$ (i.e. no substitution between attributes) and the function is of Leontief type, with L-shaped contour curves as for perfect complement attributes, hence the worst performer attribute is the only considered in the aggregator function. When $\beta=0, \sigma=1$ and $S_{i}$ is a Cobb-Douglas function, with unit substitution elasticity. Finally, when $\beta=1, S_{i}$ is a linear function of the $K$ attributes and $\sigma \rightarrow \infty$, that is, attributes are perfect substitutes, so that low levels on one of the attributes can be perfectly compensated by high levels on another. A common restriction is $\beta \leq 1$, so that the elasticity of substitution is nonnegative (hence convex to the origin contours) and the function is quasi-concave with respect to the $x_{\mathrm{s}}$, with decreasing marginal returns ${ }^{12}$. We can interpret convexity of preferences as a basic inclination of the economic agents for diversification. In other words, "the consumer prefers a more balanced (varied) bundle to a less balanced (varied) bundle" (Zamagni 1987, 139).

For the second step, Maasoumi proposes an inequality index of the GE family as a measure of the divergence between two distributions; that is, the size distribution of $S$ and the uniform distribution that has the highest entropy and represents equality of well-being.

For $\alpha \neq 0,1 \quad I_{M} \propto \begin{cases}\frac{1}{\alpha(1-\alpha)} \frac{1}{n} \sum_{i=1}^{n}\left[1-\left(\frac{\left(\sum_{k=1}^{K} w_{k} x_{i k}^{\beta}\right)^{1 / \beta}}{\bar{S}}\right)^{\alpha}\right] & , \beta \neq 0 \\ \frac{1}{\alpha(1-\alpha)} \frac{1}{n} \sum_{i=1}^{n}\left[1-\left(\frac{\prod_{k}^{K} x_{i k}^{w_{k}}}{\bar{S}}\right)^{\alpha}\right] & , \beta=0\end{cases}$

\footnotetext{
${ }^{11}$ A strong point against the CES formulation is brought forward by Bourguignon and Chakravarty (2003), . They argue that extending the measure to more attributes implies assuming that the elasticity of substitution is the same between attributes. "This may be not very satisfactory and other more complex specifications have to be designed to avoid this" (p. 40). It might be worth exploring, for instance, how to include multi-attribute elasticity of substitution (direct Allen or Morishima elasticities)

12 In the context of utility/welfare maximisation exercise, quasi-concavity of the objective function guarantees that the solution of the optimisation exercise is indeed a maximum and that the demand functions are well-behaved.
} 
For $\alpha=0 \quad I_{M}^{0}=\frac{1}{n} \sum_{i=1}^{n} \log \left[\frac{\bar{S}}{S_{i}}\right] \propto \begin{cases}\frac{1}{n} \sum_{i=1}^{n} \log \left[\frac{\bar{S}}{\left(\sum_{k=1}^{K} w_{k} x_{i k}^{\beta}\right)^{1 / \beta}}\right] & , \beta \neq 0 \\ \frac{1}{n} \sum_{i=1}^{n} \log \left[\frac{\bar{S}}{\prod_{k}^{K} x_{i k}^{w_{k}}}\right] & , \beta=0\end{cases}$

For $\alpha=1 \quad I_{M}^{1}=\frac{1}{n} \sum_{i=1}^{n} \frac{S_{i}}{\bar{S}} \log \left[\frac{S_{i}}{\bar{S}}\right] \propto\left\{\begin{array}{l}\frac{1}{n} \sum_{i=1}^{n}\left\{\frac{\left(\sum_{k=1}^{K} w_{k} x_{i k}^{\beta}\right)^{1 / \beta}}{\bar{S}} \log \left[\frac{\left(\sum_{k=1}^{K} w_{k} x_{i k}^{\beta}\right)^{1 / \beta}}{\bar{S}}\right]\right. \\ \frac{1}{n} \sum_{i=1}^{n}\left\{\frac{\prod_{k}^{K} x_{i k}^{w_{k}}}{\bar{S}} \log \left[\frac{\prod_{k}^{K} x_{i k}^{w_{k}}}{\bar{S}}\right]\right\}\end{array}, \beta=0\right.$

Where $\bar{S}=\frac{\sum_{i=1}^{n} S_{i}}{n}$ is the mean of the well-being indicator for the $n$ individuals and $\alpha$ is the 'inequality aversion' parameter as in the unidimensional formulation - the lower the $\alpha$ the more sensitive to changes at the lower part of the well-being distribution. $I_{M}$ are normalized iso-elastic transformations of the utility functions $S_{i}$. Theil's first and second indices are weighted average of attribute inequalities, reflecting the substitution effect between attributes.

It can be shown that $I_{M}$ satisfies $\mathbf{C N}, \mathbf{A N}, \mathbf{N M}, \mathbf{R I}, \mathbf{D P}$ and $\mathbf{A S}$ for all cases. The other properties can be satisfied for limited ranges of values of parameters $\alpha$ and $\beta$. SI is satisfied for all cases except when $\alpha=1$ (Theil's first measure in the univariate context ${ }^{13}$. DA holds when $\alpha=\beta$ and the SWF is individualist and specific, in Kolm (1977)'s sense ${ }^{14}$ (Proposition 1 in Maasoumi (1986)).

The two majorization criteria become rather controversial for this measure. There has been a debate over the satisfaction of $\mathbf{U M}$, which Maasoumi originally argued was always satisfied. It

\footnotetext{
${ }^{13}$ (SSI) is satisfied only when $\beta=0$ (Cobb-Douglas aggregator function) or when one works with the matrix of shares which "it requires a rather unusual assertion that individual well-being depends on shares of attributes" Maasoumi (1999), 450.. As pointed out in footnote 4, there are valid reasons to disregard this property.

${ }^{14}$ That is if it can be written as a (F) function of individual's (utility) functions [Si $\left.\left(\mathbf{x}^{i}\right)\right]$ and can be equally be written as a $(\mathrm{G})$ function of attribute's functions $\left[A_{j}\left(\mathbf{x}^{\mathrm{i}}\right)\right]$. Kolm proves that if a SEF is both individualistic and specific, it must be additive [Theorem 1, Kolm 1977].
} 
was later proved -Dardanoni (1996) provides a counterexample ${ }^{15}$ - that this is not necessarily the case, for the measure is defined with respect to the aggregate functions of the individuals, and not the actual levels of attributes (Tsui 1995). In sum, UM hold for limited ranges of value of parameters for which the $I_{M}$ is Schur-convex (or the underlying SEF Schur-concave and everywhere increasing). The reason behind this is the fact that individuals' well-being is normalised by the mean aggregator (Bourguignon 1999)16. As a result of this $I_{M}$ cannot be associated directly to a SEF (function of attributes rather than of aggregate utilities) which makes the interpretation and the finding of the condition for which UPD/UM hold quite problematic.

An additional question remains: whether and when $I_{M}$ satisfy CIM. I would argue that, yet again because of the normalisation chosen by the author, it is not clear which are the conditions for CIM to be satisfied. The reason is that, any correlation increasing transfer if preserving average for each attribute would still change the mean of well-being as defined by $\bar{S}$. This becomes apparent when obtaining the cross-partial derivative of the index with respect to two different attributes, which will depend on the way $\bar{S}$ moves after the transfer.

The two-stage approach to multidimensional inequality measures has the advantage that the aggregation of attributes - hence the SEF - is dealt directly and explicitly ${ }^{17}$. Additionally, it differs from the rest of the measures reviewed in that the basis of his measure is to be found in the theory of information rather than in the axiomatic or normative approach. Still, as the GE divergence is itself justified by a set of desirable axioms of information theory similar to those posed by the followers of an axiomatic approach, it cannot be blamed for ignoring them altogether.

\footnotetext{
15 In the example

$X=\left[\begin{array}{ccc}10 & 10 & 10 \\ 10 & 90 & 10 \\ 90 & 10 & 10\end{array}\right], B=\left[\begin{array}{ccc}1 & 0 & 0 \\ 0 & 0.5 & 0.5 \\ 0 & 0.5 & 0.5\end{array}\right]$ so that $Y=X B=\left[\begin{array}{ccc}10 & 10 & 10 \\ 50 & 50 & 10 \\ 50 & 50 & 10\end{array}\right]$
}

Dardanoni (1996), shows that applying any concave $S_{i}$ any Schur-convex inequality index will increase after the 'equalising' transformation. He also offers a second criticism to Maasoumi two-step approach and, in particular, a criticism to the (UPD) as a valid majorization criterion. He argues that "while having a clear interpretation in terms of social welfare, is uninformative for evaluating the amount of inequality in the society" (Dardanoni (1996), 202.. In exchange, he offers a new majorization, the so called "unfair redistribution' (UR) which, in his view is a mild requirement. Maasoumi responds by pointing out that (UR) assumes that all attributes are valued equally and are perfects substitutes $(\beta=1)$, and thus, $S_{i}$ functions are additive separable (AS). He discards this majorization criterion (even if it might be desirable in theory) because it finds the assumption of perfect substitutability of attributes rather restricted. This is also supported by Savaglio when he argues that "it is in contradiction with the evaluation of individual welfare when there are correlations between attributes of a distribution" (Savaglio (2006)). A similar argument was brought by Bourguignon and Chakravarty (2003) in their critique to Tsui CIM majorization.

16 Also, because of this normalisation it does not avoid double-counting, as argued in his text.

17 According to Maasoumi "there is an inevitable aggregation of the $[\mathrm{K}]$ attributes that will result in any scalar measure" Maasoumi (1999), 447. but this inevitability is questioned when aggregation is done in the first stage across individuals (Gajdos and Weymark (2005), ) or when proposing non-decomposable multidimensional inequality indices (Tsui (1999), ). 


\subsection{Tsui $(1995,1999)$}

In his two papers on multidimensional inequality indices, Tsui derives through an axiomatic approach, a set of admissible classes of SEF and corresponding inequality indices - both relative and absolute. In here, I will focus on one of the family of relative measures, which satisfies the most properties and majorization criteria.

After defining a set of desirable axioms on the SEF18, Tsui (1995) derives a valid $W(X)$ which is ordinally equivalent to $\sum_{i} u\left(\mathbf{x}^{\mathrm{i}}\right)$ where $u($.$) is a strictly increasing concave function of the$ forms $u\left(\mathbf{x}^{\mathrm{i}}\right)=a+b \prod_{k=1}^{K} x_{i k}^{r_{k}}$ and where $r_{k}$ and $b$ are restricted so that the functions are increasing and strictly concave. Utilising Kolm's relative inequality index derivation, Tsui proposes the following multidimensional inequality index ${ }^{19}$ :

$$
I_{T R 1}=1-\left[\frac{1}{n} \sum_{i=1}^{n} \prod_{k=1}^{K}\left(\frac{x_{i k}}{\mu_{k}}\right)^{r_{k}}\right] / \sum_{k=1}^{K} r_{k}
$$

Tsui (1999) generalises the previous index in such a way as to satisfy both UPD/UM and CIM majorization criteria, while relaxing the property of AS to a less strict one of $\mathrm{D}^{20}$. These, together with the other generally agreed set of axioms lead inexorably to the class of multi-attribute generalized entropy measures of the following form

$$
F\left(I_{T 1}(X)\right)=\frac{\rho}{n} \sum_{i=1}^{n}\left[\prod_{k=1}^{K}\left(\frac{x_{i k}}{\mu_{k}}\right)^{c_{k}}-1\right]
$$

\footnotetext{
18 These are continuity, strictly increasing with the elements of $\mathrm{X}$, anonymity, strictly quasi-concave, decomposability, and ratio-scale invariance.

${ }^{19}$ The other relative index proposed also is based on an individual Cobb-Douglas function but aggregation across persons is done through a SEF of the Nash type, that is a product of individuals' well-being; Tsui (1995), .

${ }^{20}$ On this the author already in 1995 noticed that "Additive separability is considered by some as rather restrictive ... In the final analysis, whether a separability axiom of some sort is acceptable cannot be resolved by purely logical arguments, as are other axioms in this paper. There is also a priori no reason why Ax 5 may not be discarded. It follows that one may have sets of axioms without Ax 5 and yet derive the corresponding classes of social evaluation functions and inequality indices [...] It is my conjecture that replacing Ax 5 by a decomposability axiom in Shorrocks will lead to a class of non-separable social evaluation functions embedding the class of multidimensional GE measures [introduced in 1999]. In other words, an aggregate function of attributes for each person is not always a necessary first step to the design of reasonable multidimensional indices" [(1995), 253.]
} 


$$
F\left(I_{T 2}(X)\right)=\frac{1}{n} \sum_{i=1}^{n}\left(\frac{x_{m i}}{\mu_{m}}\right)\left[\sum_{k=1}^{K} w_{m j} \log \left(\frac{x_{i k}}{\mu_{k}}\right)\right]
$$

where $m \in\{1,2, \ldots, K\}$

$$
F\left(I_{T 3}(X)\right)=\frac{1}{n} \sum_{i=1}^{n} \sum_{k=1}^{K} \delta_{k} \log \left(\mu_{k} / x_{i k}\right)
$$

Where $F()$ is an strictly increasing function of $I^{n}()$, and value zero when $I^{n}()=0$.

These indices satisfy $\mathbf{C N}, \mathbf{A N}, \mathbf{N M}, \mathbf{D}, \mathbf{R I}$, and $\mathbf{R S}$. UM and UPD are satisfied whenever the (individual) function is strictly convex, hence restricting the parameters so that the corresponding Hessian matrix is positive semidefinite. In the case of [7], although the underlying individual function is similar to Maasoumi's, UPD is satisfied because the normalisation by the 'well-being' of the average person endowed with the average level for each attribute instead of by the mean of individual utility. It is because of this different normalisation that $I_{T 1}$ is free from Dardanoni's critique mentioned earlier.

Similar to Maaosumi, CIM will be satisfied under specific values of the parameters. The majorization criterion will be restricted to strictly L-superadditive ${ }^{21}$, which is equivalent to the second partial derivative of the inequality index with respect to attributes to be positive - a similar condition already mentioned in Atkinson and Bourguignon (1982) though stated in terms of the utility function, hence with opposite sign. In terms of the indices proposed above, it will eliminate [8] and [9], leading to focus on [7] whenever $\varrho c_{i} j>0$, for $i, j=1,2, \ldots, K$.

The main criticism of Tsui formulation is that it lacks an explicit exploration of the meaning of the parameters in the formulas, making it particularly hard to disentangle their roles as weights, degree of substitution between attributes, and inequality aversion, and in fact, mixing them. This will become problematic when, in practice, one wants to specify the reasonable ranges of values of parameters.

To see this more clearly we can use the Atkinson-Kolm normative approach relating social evaluation functions and relative inequality indices (Atkinson 1970; Kolm 1969). In an inversed process as utilised in Tsui (1995), we can derive from [7] the underlying SEF.

\footnotetext{
${ }^{21}$ i.e. $\phi(\mathbf{x} \wedge \mathbf{y})+\phi(\mathbf{x} \vee \mathbf{y})>\phi(\mathbf{x})+\phi(\mathbf{y})$ if $\mathbf{x} \wedge \mathbf{y} \neq \mathbf{x}$ or $\mathbf{y}$ or equivalently $\phi_{k m}=\frac{\partial^{2} \phi}{\partial x_{k} \partial x_{l}}>0, k, l=$ $1,2, \ldots, K, k \neq l$.
} 


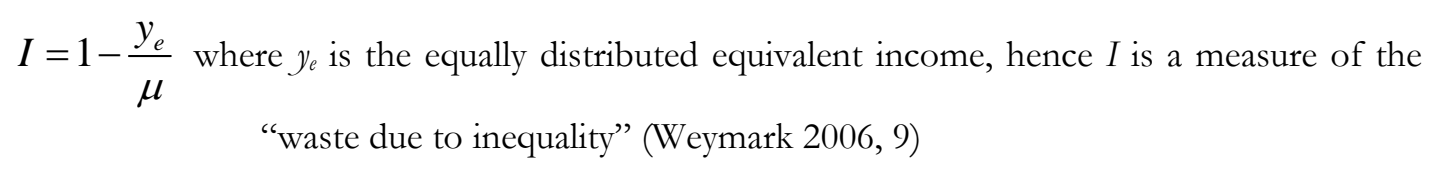

If the SEF takes the standard form of the sum of individuals' well-being, then

$$
W=n y_{e}=\mu(1-I)
$$

Rearranging [7] it can be expressed as

$$
F\left(I_{T 1}(X)\right)=(-\rho)\left[1-\frac{\left[\sum_{i=1}^{n} \prod_{k=1}^{K} x_{i k}{ }^{c_{k}}\right] / n}{\prod_{k=1}^{K} \mu_{k}^{c_{k}}}\right]
$$

Then individual well-being is of the form $\prod_{k=1}^{K} x_{i k}{ }^{c_{k}}$ which is a traditional Cobb-Douglas function, with constant elasticity of substitution, equal to one $(\beta=0)$. Inequality is constructed as the aggregation of individuals with respect to their shortfall to the 'utility' of a fictitious individual endowed with the average level of all attributes, using and additive SEF. It is straightforward to see in [7] that [6] is obtained when $\rho=-1, \mathrm{~F}()=\mathrm{I}(), \Sigma_{c_{k}}=1$ and thus, $c_{k}=r_{k}$.

The parameter $c_{k}$ represents both the weight and the inequality aversion component of the index. From the individual utility function, we see that $c_{k}$ determines the contribution (weight) of each attribute to the individual well-being. That is, how much the well-being increases or decreases with the increase of the attribute (first derivative), and the concavity of the function (i.e. second derivative) measuring the rate of return of each attribute. In this case, a logical restriction will be to set $c_{k}$ to be positive but less than $1:$ then,

$$
0<c_{k}<1 \quad \text { will be the first boundary to the parameter. }
$$

On the other hand, in the inequality index [7] $c_{k}$ plays the role of an inequality aversion parameter, that is, the different weights to changes occurred at different parts of the distribution (seen in the sign of the cross-partial derivative with respect to individuals for any one attribute, comparing that of a well-off and a less well-off person). As already mentioned, CIM will also restrict the admissible values of parameters so that $\varrho c_{m} c_{l}>0$ is satisfied for any $m, l=1,2, \ldots K .{ }^{22}$

\footnotetext{
${ }^{22}$ In the literature, critiques of these indices proposed by Tsui come from two main lines. Weymark argues that none of these indices are provided with a social evaluation foundation as the axiomatisation is done directly rather than through a social evaluation function (Weymark (2006), ). Even if not done explicitly in
} 


\subsection{Bourguignon (1999)}

In his comment to Maasoumi (1999)'s article Bourguignon proposes a multidimensional inequality measure which provides a more direct link with (standard utilitarian) SEFs than $I_{M}$, hence also with multidimensional stochastic dominance criteria (Kolm 1977, AB1982). The normalisation used in the inequality measure is similar to Tsui's hence satisfying similar properties as there.

In the same spirit as suggested in 1982, Bourguignon bases the inequality index on an individual cardinal function representing the ordinal preferences embedded. This is an strictly monotone and concave function of the form

$$
S(X)=\left(\sum_{k=1}^{K} w_{k} x_{i k}^{\beta}\right)^{\alpha / \beta}
$$

where $\alpha$ is related to the degree of aversion of the society with respect to inequality and the degree of substitution is normally set to be $\beta \leq 1$. The related multidimensional inequality measure proposed is a natural extension of a Dalton measure

$$
I_{B}=1-\frac{1}{n} \frac{\sum_{i}\left[\sum_{k=1}^{K}\left(w_{k} x_{i k}^{\beta}\right)\right]^{\alpha / \beta}}{\left[\sum_{k=1}^{K}\left(w_{k} \mu_{k}^{\beta}\right)\right]^{\alpha / \beta}}
$$

Maximum social welfare (this understood as the sum of individual welfares) is achieved with perfect equality, giving to each person the average quantity of each attribute.

If we allow for $\beta$ to be equal to zero, we can present a family of multidimensional inequality indices, which includes Tsui's measures. The class of indices will be

Tsui paper, it is possible to derive the underlying SEF using Atkinson-Kolm approach, as shown here. List instead would focus on the fact that the indices ignore interpersonal comparisons of well-being when using a "version of the somewhat controversial axiom of (additive) decomposability, which, by requiring us to ignore some - arguably useful - information in a distribution, is known to rule out all but entropy-based measures in various economic and information-theoretic contexts" (List (1999), 3.. In this case, one should restrict the attention to Gini family of indices. This is a valid point, which deserves further exploration Anderson (2004), , List (1999), , Weymark (2006), . 
when $\alpha \neq 0$

$$
\begin{aligned}
& F\left(I_{B}\right)=(-\rho)\left[1-\frac{1}{n} \frac{\sum_{i}\left[\sum_{k=1}^{K}\left(w_{k} x_{i k}^{\beta}\right)\right]^{\alpha / \beta}}{\left[\sum_{k=1}^{K}\left(w_{k} \mu_{k}^{\beta}\right)\right]^{\alpha / \beta}}\right] \text { when } \beta \neq 0 \\
& F\left(I_{B}\right)=(-\rho)\left[1-\frac{1}{n} \frac{\sum_{i}\left[\prod_{k=1}^{K} x_{i k}^{w_{k}}\right]^{\alpha}}{\left[\prod^{K} \mu_{k}\right.}\right] \quad \text { when } \beta=0
\end{aligned}
$$

$\underline{\text { when } \alpha=0}$

$$
\begin{aligned}
& F\left(I_{B}\right)=(-\rho)\left[\frac{1}{n} \sum_{i=1}^{n} \log \left(\frac{\sum_{k=1}^{K} w_{k} \mu_{k}^{\beta}}{\sum_{k=1}^{K} w_{k} x_{i k}^{\beta}}\right)^{1 / \beta}\right] \quad \text { when } \beta \neq 0 \\
& F\left(I_{B}\right)=(-\rho)\left[\frac{1}{n} \log \left(\frac{\prod_{k=1}^{K} \mu_{k}^{w_{k}}}{\prod_{k=1}^{K} x_{i k}^{w_{k}}}\right)\right]
\end{aligned}
$$

where $\varrho$ is set so that $F()$ is an increasing and concave function of $I_{B}$ and $F()$ takes value zero when $I_{B}=0$ [14] resembles directly Tsui's measures whenever $c_{k}=\alpha w_{k}$ It is now clearer that the $c_{k}$ parameters in [7] is in fact, made of two components: one related to the degree of inequality aversion and another reflecting each attributes contribution to total well-being.

Bourguignon's index is related to the Maasoumi's but, at the same time, "significantly different". The $I_{B}$ has embedded an aggregator that is a distance function. In this respect, it is similar to Maasoumi's. However, in $I_{B}$ individual welfare is normalised - in a similar fashion to Tsui (1995) - using the value of the aggregator for the mean individual; i.e. the one endowed with mean attributes $\left[\sum_{k=1}^{K}\left(w_{k} \mu_{k}^{\beta}\right)^{1 / \beta}\right]$, whereas in $I_{M}$ normalisation is done by the mean aggregator 


$$
\left[\sum_{i=1}^{n}\left(\sum_{k=1}^{K} w_{k} X_{i k}^{\beta}\right)^{1 / \beta} / n\right] 23 \text {. It is, in fact, due to this different normalisation that is possible to }
$$

study when $I_{B}$ satisfies desirable majorization criteria, while the issue remains uncertain for $I_{M}$.

$I_{B}$ satisfies $\mathbf{C N}, \mathbf{A N}$, and $\mathbf{N M}$. Furthermore, the measure is consistent with UPD whenever $\beta<$ 1 and $\alpha<1$ and $\beta$ is below some level increasing with $\alpha$. SI is satisfied whereas SSI is satisfied only when $\beta=0$ (Cobb-Douglas SEF) which is, in itself, quite restrictive. Still, Bourguignon argues that not satisfying this property is in fact an interesting feature of the measure in the context of multidimensional inequality. As with $I_{\mathrm{M}}$, it is straightforward to show that DA holds when $\alpha=\beta$.

Finally, we can show that CIM holds whenever $(\alpha<\beta \text { and } \alpha<1)^{24}$ while $\alpha>0$. That is, for this property to be satisfied the parameters of substitutability between attributes and inequality aversion should maintain a specific relation. As indicated by Bourguignon (1999) this is a clear advantage of CES-like forms as the condition for inequality increase (decrease) after a correlation increasing but attribute mean preserving transfer can be expressed in terms of the partial crossderivative of the inequality index which, in this case, is a function of the parameters used in the specification of the SEF. This was already highlighted in Atkinson and Bourguignon (1982)25. In other words, for an index to remain CIM lower substitutability between attributes has to be accompanied by a higher sensitivity to the lower part of the distribution.

It is obvious that the DA condition $(\alpha=\beta)$ is inconsistent with CIM for $I_{B}$ as both cannot hold simultaneously. Therefore, if we are working with aggregate data such as described previously, we must restrict $I_{B}$ to this special case for which neither CIM nor its contrary (as proposed by Bourguignon) are satisfied. Rather, $I_{B}$ will not experience any change in the measurement with correlation increasing but invariant mean transfers.

${ }^{23}$ We can express $I_{B}$ as a linear transformation of $I_{M} \cdot I_{B}=\left[\alpha(1-\alpha) I_{M}-1\right] R^{\alpha}+1=\left(1-R^{\alpha}\right)+\alpha(1-\alpha) R^{\alpha} I_{M}$ where $R=\frac{\sum_{i=1}^{n}\left(\sum_{k=1}^{K} w_{k} x_{i k}^{\beta}\right)^{1 / \beta}}{\left(x^{1 / \beta}\right.}$ is the 'corrective term' which is the ratio between the mean aggregator and the $n\left(\sum_{k=1}^{K} w_{k} \mu_{i k}^{\beta}\right)^{1 / \beta}$

aggregator at the mean Because of this term, Bourguignon argues that Maasoumi's measure cannot be directly related to a social welfare function, "which makes it difficult to interpret" Bourguignon (1999), 478.. [NB: different from Bourguignon's formula. I thank Koen Decancq for this correction].

${ }^{24}$ Strictly speaking, also $(\alpha>\beta$ and $\alpha>1)$ is a condition for CIM but it is not consistent with previous restrictions

25 "Where the preferences are homothetic, but we allow concave transformations, then the sign of $\mathrm{v}_{12}$ depends on whether the degree of relative risk aversion $[\alpha]$ is greater or less than $1 / \sigma$ " (Atkinson and Bourguignon (1982), 191., where $\sigma$ is the standard definition of the elasticity of substitution. 
We have shown that once we extend Bourguignon index to include unit elasticity of substitution, we arrive to a family of indices which has both the advantage of satisfying a set of desirable properties and majorization criteria proposed in the literature and explicitly distinguishes the different roles of the parameters included in the index.

\section{Application to the Argentina data}

In this section we present an application of the multidimensional inequality measures to the Argentine data. In particular, we will compare 1991 and 2001 individuals' well-being as described (quite restrictively) by three dimensions: income, health, and education. Other than these, individuals are assumed to be homogeneous in all other relevant characteristics. The present exercise follows from a previous paper (Lugo, 2004) where provincial distributions were utilised. A great limitation of that work was that it ignored the within province inequality, which usually account for most part of the total inequality for any of the attributes considered. The main reason behind the choice was data limitations. In the present exercise we use all available information at the level of individuals, coming from Permanent Household Survey, Vital Statistics and National Population Censuses.

We utilise household survey data, focusing on adult population living in the 25 most populationwise important cities of the country. The indicators utilised are real per capita bousehold income, life expectancy at birth (as given by gender and city), and years in the formal educational system (see Annex).

During the 1990s Argentina went through a process of adjustment, stabilization, rapid output expansion, and a final collapse of the economy. In clear contrast with previous decades, in the first part of the 1990s, Argentina enjoyed fast economic growth and a drastic fall in inflation rates (Frenkel 2002; Heymann 2000). After a series of financial crises - Russia 1998, Brazil 1999 followed by a substantial decline in private capital inflows, Argentina entered a recession from which only five or six years after, does it seem to be recovering ${ }^{26}$. The drastic movements in the country's economic activity will, not surprisingly, have an effect on the level and distribution of the population's well being. The 1990s represented a period of continuous deterioration of labour market conditions and increase in income poverty and inequality levels. In the period, the unemployment rate tripled while income inequality and poverty rates increased to levels of unprecedented magnitude (Altimir et al. 2002; Gasparini 2002; Gonzalez Rozada and Menendez

\footnotetext{
${ }^{26}$ Other reasons behind the collapse of the economy relate to the continuous appreciation of the currency (Frenkel and Gonzalez Rozada (2000), , Heymann (2000), ) and the existence of macroeconomic policy inconsistencies Blejer (2002), .
} 
2002). At the same time, health and education as measured by basic indicators continued with the positive trends from the previous decades ${ }^{27}$.

Between 1991 and 2001, on average, household per capita income decreased slightly whereas life expectancy and years of education both showed an average progress (Table A.1 Statistical Appendix). Correlation between income and education almost doubled while income and health and health and education were in both years significantly low. This is largely due to the nature of the data used, as a large part of the variation in health between individuals is being ignored (see Annex for explanation of the indicators). Finally, distribution among individuals as measured by the Gini coefficient and several GE indices worsened for income while improved for health and education (Table A.2). It is because of this different behaviour of the attributes (in both means and distributions) that the question on how we measure multivariate inequality becomes particularly relevant.

\subsection{Multidimensional inequality measurements}

Originally, real PCHI is measured in money units, life expectancy at birth in years, and education also in year of completed education. Hence, the first step into the use of an aggregate index of well-being inequality implies choosing a way of transforming all variables so as have the same unit of measurement. Naturally, the manner in which the transformation is performed will affect the results of the analysis. Human Development Index relies on agreed goalposts (minimum and maximum) and arrives at indices that fall between 0 and 1 (UNDP 1995); others, such as Hirschberg et al $(1991,2001)$ prefer variable standardisation -using the variable mean and variance $^{28}$. In the present exercise we use the HDI approach, i.e. a simple average of the three indices. We apply the following general formula:

\footnotetext{
27 The positive performances during the decade of health and education indicators should be taken with care. Both life expectancy at birth and literacy rate are variables that tend to be rather stable and have a natural upward tendency. For a comprehensive understanding of achievements in these two dimensions we should complement the study with other indicators (e.g. avoidable deaths infant mortality rate, repetition and drop-out rates, assessment of education results). UNICEF (2002) opposes the improvements in IMR and life expectancy indicators with other less positive performances observed throughout the 1990s. In particular, the report emphasises the stagnation of the rate of reduction of the IMR, the constant high proportion of infant deaths that could be avoided though low cost interventions, and the increasing regional disparities of infant mortality rates. Within education achievements, school quality assessment tests show a decline in the performance of students in secondary education in the last five years of the decade Unfortunately, these sorts of assessments were systematically performed in all provinces only since 1997.

${ }^{28}$ Hirschberg, et al. (2001), opt for rescaling each attribute to have a mean of zero and a variance of one. No log transformation is made to income. This procedure of standardising the variables will result in indices for each dimension that have positive as well as negative values, which might be problematic when using inequality measures.
} 


$$
S_{i k}=\frac{x_{i k}-\min x_{i k}}{\max x_{i k}-\min x_{i k}} \text { with } i=1, \ldots, 23 \text { and } k=h, e, y
$$

As the purpose is to compare 1991 and 2001 distributions, the goalpost will be identical for both distributions, chosen as the minimum and maximum values across both years. The minimum value of health is achieved in 1991 and its maximum in 2001, while the opposite is true for income. In education, the variable is bounded by definition ( 0 to 17$)$.

We now need to set values for the parameters involved in the multidimensional indices. To simplify the presentation we utilise equal weight $\left(w_{1}=w_{2}=w_{3}=w_{k}=1 / 3\right)$ and a range of both degrees of substitution and inequality aversion parameter for which reasonable boundaries are set normatively [ $\beta$ was set less than 1 so that $\sigma$ is greater than zero; $\alpha$ is limited to values smaller than one]. In particular, we present results for $\beta=[-20 ;-4 ; 0 ; 0.5 ; 1]$ and $\alpha=[-1 ; 0 ; 0.333 ; 0.5 ; 1]$. The Tsui index belongs to the Bourguignon family when $\beta=0, c_{k}=\alpha w_{k}$, and $\varrho=-1$.

Table 1 presents results, with different shadings to distinguish cases in which inequality in 1991 was higher than in 2001 (dark grey), there is no differences in the measurement (light grey), and 2001's inequality measurement was higher than in 1991 (white). It is apparent that there is no unambiguous conclusion over whether there was an improvement or worsening of the well-being distribution across individuals in Argentina between these two years. The conclusion will depend on the combination of $\alpha$ and $\beta$ selected.

Table 1. Multidimensional Inequality Index

Adults (aged 25 and over), Argentina 1991 and 2001

$1991>2001$ $1991=2001$

$1991<2001$

\begin{tabular}{|c|c|c|c|c|c|c|c|c|c|c|c|c|}
\hline & \multicolumn{12}{|c|}{ Degree of substitution (inv related to elastiticity of subst) } \\
\hline & \multicolumn{2}{|c|}{$\beta=-20$} & \multicolumn{2}{|c|}{$\beta=-4$} & \multicolumn{2}{|c|}{$\beta=0$} & \multicolumn{2}{|c|}{$\beta=0.333$} & \multicolumn{2}{|c|}{$\beta=0.5$} & \multicolumn{2}{|c|}{$\beta=1$} \\
\hline & 1991 & 2001 & 1991 & 2001 & 1991 & 2001 & 1991 & 2001 & 1991 & 2001 & 1991 & 2001 \\
\hline \multicolumn{13}{|c|}{$\begin{array}{c}\text { Inequality aversion parameter } \\
\text { Maasoumi }\end{array}$} \\
\hline$\alpha=-2$ & 1.653 & 22.748 & 1.645 & 22.651 & 0.103 & 0.157 & 60.554 & 0.286 & 9.228 & 0.153 & 1.953 & 0.102 \\
\hline$\alpha=-1$ & 0.426 & 0.984 & 0.424 & 0.981 & 0.079 & 0.115 & 0.258 & 0.120 & 0.156 & 0.092 & 0.112 & 0.075 \\
\hline$\alpha=0$ & 0.296 & 0.452 & 0.295 & 0.450 & 0.057 & 0.093 & 0.100 & 0.085 & 0.087 & 0.073 & 0.075 & 0.063 \\
\hline$\alpha=0.333$ & 0.279 & 0.415 & 0.277 & 0.413 & 0.180 & 0.153 & 0.092 & 0.080 & 0.081 & 0.070 & 0.071 & 0.060 \\
\hline$\alpha=0.5$ & 0.267 & 0.403 & 0.265 & 0.400 & 0.150 & 0.137 & 0.089 & 0.078 & 0.079 & 0.069 & 0.069 & 0.059 \\
\hline \multirow[t]{3}{*}{$\alpha=1$} & 0.394 & 0.470 & 0.390 & 0.466 & 0.121 & 0.122 & 0.083 & 0.075 & 0.075 & 0.066 & 0.065 & 0.057 \\
\hline & \multirow{2}{*}{\multicolumn{4}{|c|}{ Bourguignon }} & & & & & & & & \\
\hline & & & & & \multicolumn{2}{|c|}{ Tsui 99} & & & & & & \\
\hline$\alpha=-2$ & -10.914 & -139.205 & -10.915 & -139.205 & -1.141 & -1.545 & -429.87 & -2.123 & -62.034 & -1.097 & -11.716 & -0.612 \\
\hline$\alpha=-1$ & -0.905 & -1.982 & -0.906 & -1.982 & -0.310 & -0.395 & -0.649 & -0.330 & -0.388 & -0.238 & -0.225 & -0.150 \\
\hline$\alpha=0$ & -0.203 & -0.229 & -0.203 & -0.229 & -0.203 & -0.229 & -0.203 & -0.229 & -0.203 & -0.229 & -0.203 & -0.229 \\
\hline$\alpha=0.333$ & 0.106 & 0.110 & 0.106 & 0.111 & 0.087 & 0.077 & 0.047 & 0.040 & 0.036 & 0.030 & 0.016 & 0.013 \\
\hline$\alpha=0.5$ & 0.118 & 0.120 & 0.118 & 0.121 & 0.107 & 0.099 & 0.062 & 0.053 & 0.047 & 0.039 & 0.017 & 0.015 \\
\hline$\alpha=1$ & 0.044 & 0.010 & 0.046 & 0.012 & 0.139 & 0.129 & 0.080 & 0.068 & 0.054 & 0.044 & 0.000 & 0.000 \\
\hline
\end{tabular}

Source: author's calculation

Note: variables included in the index are per capita household income, life expectancy at birth, and years of completed formal education. Common measurement transformation was done following HDI approach of max and min goalpost. 
It is interesting that for Maasoumi's index, $\beta$ was the only parameter relevant for the conclusion that is, irrespectively of the value chosen for $\alpha, 1991$ presents lower inequality measurement than 2001 when $\beta$ is set less or equal to zero, whereas the contrary in true for positive degrees of substitutions. On the other hand, when applying Bourguignon family of measures, results are more mixed, showing the interplay between the two parameters $\alpha$ and $\beta$

Understandably, one could be still rather disappointed by an ambiguous conclusion as the one found. Instead, we can utilise the previous analysis of conditions for satisfaction of properties and majorization criteria to define "preferred ranges" of parameters, thus restricting the space of the table where to focus. Now it becomes evident the advantage of having a measure where it's properties are directly related to the value of parameters, such in the case of Bourguignon index. In particular, UPD will restrict $\beta<1$ and $\alpha<1$ while CIM would make us focus on the cells where $\alpha<\beta$ and $\alpha>0$. In Table 1 the above restriction will force us to focus on the pair $[\alpha=0.333 ; \beta=0.5]$ for which 1991 inequality measurement is greater than 2001. If instead, one were to prefer the opposite of CIM - as also argued by Bourguignon to be valid when attributes are complement - we should focus on the pairs for which $\alpha>\beta$ and $0<\alpha<1$ we obtain the opposite results, that is, 2001 presents higher multidimensional inequality of well-being measurement than 199129. Unfortunately, we haven't yet been able to identify (if possible) similar conditions for Maasoumi index.

\section{Conclusion}

The multidimensional inequality index proposed by Maasoumi has the noteworthy feature that it is clear and explicit in the role of parameters involved in the formula, hence making it easier to set reasonable (ranges of) values for each case. It distinguishes the different elements involved in any multivariate function of inequality. On the other hand, it is yet not clear when it satisfies a desirable set of properties and majorization criteria. Tsui suggested several inequality indices which, by construction, allow us to identify directly the value judgement involved in the selection of options at hand ${ }^{30}$. The main limitation of Tsui presentation is that it does not allow a direct disentangling of the meaning of parameters, hence complicating the setting of reasonable boundaries. Bourguignon combines the advantageous characteristics of both previous authors. In the present paper we extend Bourguignon formulation so as to include Tsui's measures, while

\footnotetext{
${ }^{29}$ Naturally, we would need to compute confidence intervals for each measurement to be sure whether the differences are statistically significant. The point of the exercise in this paper was merely illustrative, but it would certainly need doing for a proper comparison of two distributions. As the distribution is now know, a method of bootstrapping can be employed.

30 "In the final analysis, it is unlikely to have a set of axioms that command a consensus since the problem at hand is inherently ethical. What seems more important is complete characterization of social evaluation functions and the corresponding inequality indices so that the users of these indices will have a clear picture of the ethical judgements implicit in them"; Tsui (1995), 261.
} 
allowing for different elasticities of substitution between attributes (even if constant for all pairs) and distinguishing the weights, on the one hand, and degree of inequality aversion, on the other. It also allows for a clear setting of conditions related to desirable majorization criteria, making more transparent the implications of the choice of values of parameters.

Maasoumi's proposal whilst offering an appealing method to form inequality measures, in which all the elements of the functions and their roles are explicitly considered, suffers from the weakness that it yet not clear when or whether the principle of transfers and correlation increasing majorization are satisfied. Tsui propose various sets of measures encompassing this problem. On the other hand, Tsui falls short on the interpretation of the parameters involved in the inequality measures, which does not allow an easy disentangling of their meaning. Bourguignon presents an index dealing with some of these aspects. Some of the weaknesses of Maasoumi remain.

More research in the area remains to be done. Among other areas of further research we should pursue a similar study of the multidimensional indices of the Gini family already present in the literature (Anderson 2004; Gajdos and Weymark 2005; Koshevoy and Mosler 1997; List 1999; Weymark 2006). Furthermore, it will be worth examining whether it is possible and how to include different elasticities of substitution for each pair of attributes and/or different inequality aversion parameters. 


\section{Statistical Appendix}

Table A.1. Basic summary statistics of indicators used Adults (aged 25 and over), Argentina 1991 and 2001

\begin{tabular}{|c|c|c|c|c|c|c|c|c|c|}
\hline \multirow[b]{2}{*}{ year $=1991$} & \multirow[t]{2}{*}{ Obs } & \multicolumn{2}{|c|}{ Mean Std. Dev } & \multirow[t]{2}{*}{ Min } & \multirow[t]{2}{*}{ Max } & \multicolumn{4}{|c|}{ Pearson correlation coefficients } \\
\hline & & & & & & & & eduyears & rrpchi \\
\hline ex & $8,224,879$ & 73.0 & 4.0 & 65.6 & 79.0 & ex & 1.00 & & \\
\hline eduyears & $8,213,310$ & 9.1 & 4.4 & - & 17.0 & eduyears & -0.07 & 1.00 & \\
\hline rrpchi & $8,224,879$ & 239.9 & 356.1 & 2.1 & $11,250.0$ & rrpchi & 0.00 & 0.25 & 1.00 \\
\hline year $=2001$ & & & & & & & & eduyears & rrpchi \\
\hline ex & $10,544,339$ & 73.9 & 3.9 & 67.0 & 79.9 & ex & 1.00 & & \\
\hline eduyears & $10,540,395$ & 9.7 & 4.2 & - & 17.0 & eduyears & 0.03 & 1.00 & \\
\hline rrpchi & $10,544,339$ & 238.8 & 290.7 & 1.3 & $7,497.9$ & rrpchi & 0.02 & 0.42 & 1.00 \\
\hline
\end{tabular}

Source: author's calculation, based on PHS (October spells), Vital Statistics, and National Population Censuses

where EX: life expectancy at birth (in years)

EDUYEARS: years in the formal educational system

RPCHI: Real per capita household income (monthly, pesos\$)

Table A.2. Inequality for each indicator

Adults (aged 25 and over), Argentina 1991 and 2001

$1991>2001 \quad 1991=2001 \quad 1991<2001$

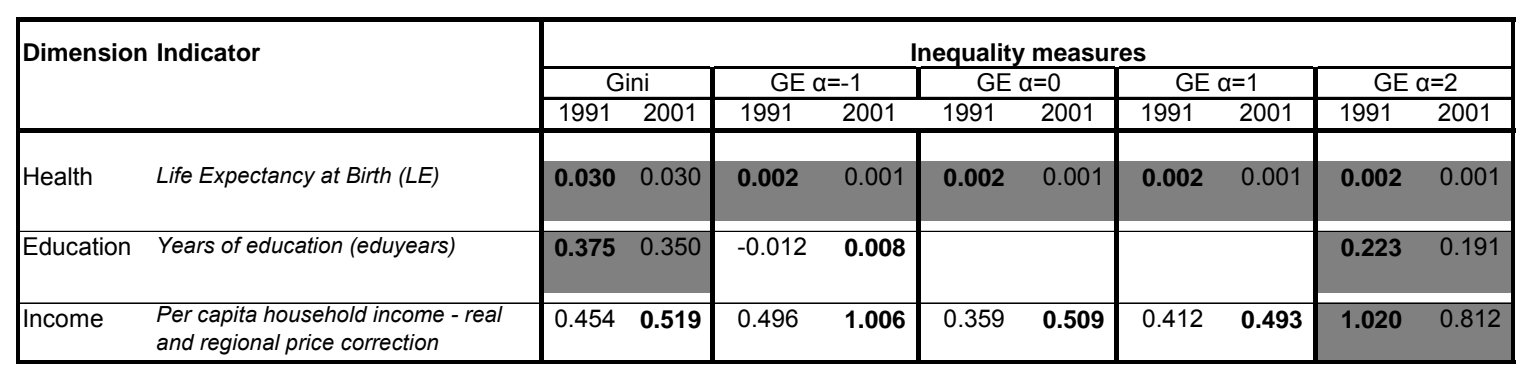




\section{Annex. The Data}

- Income: per capita household income ${ }^{31}$. Source: Permanent Household Survey, October spells. We adjust incomes for inflation. Unfortunately, it can only be done using the Greater Buenos Aires Consumer Price Index as no other option is available ${ }^{32}$. A more serious limitation of the present data is the lack of an index to adjust for regional price differentials. Indeed, different regions face different prices for goods and services, hence affecting individuals' ability to convert those incomes onto goods. Throughout the 1990s prices have not been systematically surveyed in all provinces but only in Greater Buenos Aires. The 1996 National Household Consumption Survey provides a price index for a basket of goods and services for each of the country's six regions ${ }^{33}$. No other alternative is available at present and we apply it for both beginning and end of the period. Hence, we implicitly assume that the regional price differential in 1991 is the same as in 1997 and 2001, certainly a very questionable assumption.

- Health: Life expectancy at birth: average number of years a newborn person is expected to live if he/she passes through life subject to the age-specific death rates of a given period. It is the sum of the mortality rates for all ages combined, expressed in years. As life expectancy is an aggregate variable (not individual) we assigned to each adult the life expectancy corresponding to his group according to gender and city. Sources: life tables from Vital Statistics (Ministerio de Salud). Population per age groups from Population Censuses. Given the different definition of the cities used in PHS and Vital Statistics and Population Censuses, life expectancy was computed for the departments to which the cities belong.

- Education: Years of formal education. Sources: Permanent Household Survey, October spells. As declared by the individual.

\footnotetext{
${ }^{31}$ We don't include household with incomplete or zero responses. This might bring some uncertainty as they are not distributed homogeneously across cities and has changed over time. In 1991, 22\% of the households have incomplete incomes, while in 2001 the proportion is $12 \%$, , hence not only significant but also changing over time.

32 Strictly speaking, comparisons of mean levels of incomes are relevant if the interest is on welfare instead of solely inequality. Scale independence of inequality measures is a desirable characteristic when studying inequality, i.e. invariant with respect to proportional shifts in the distributions.

${ }^{33}$ These are the regional price differentials used by the official institution to calculate regional poverty lines.
} 


\section{Reference:}

Altimir, O., L. Beccaria, and M. Gonzalez Rozada. (2002), "La distribución del ingreso en Argentina, 1974-2000", Revista de la CEPAL, 78: 55-85.

Anderson, G. (2004), "Indices and Tests for Multidimensional Inequality:

Multivariate Generalizations of the Gini Coefficient and Kolmogorov-Smirnov TwoSample Test" presented at the $28^{\text {th }}$ General Conference of the International Association for Research in Income and Wealth, Cork, Ireland: IARIW.

Atkinson, A. B. (1970), "On the Measurement of Inequality", Journal of Economic Theory, 2 (3): 244-263.

Atkinson, A. B., and F. Bourguignon. (1982), "The Comparison of MultiDimensioned Distributions of Economic Status", Review of Economic Studies, 49 (2): 183-201.

Blejer, M. (2002), "The Role of Central Banks in Financial Crises" presented at the 9th Conference on central banking, Washington, DC.

Boche, H., and E. Jorswieck. (2004), "On Schur-convexity of expectation of weighted sum of random variables with applications", Journal of Inequalities in Pure and Applied Mathematics, 5 (2): 1-14 (article 46).

Boland, P. J., and F. Proschan. (1988), "Multivariate arrangement increasing functions with application in probability and statistics", Journal of Multivariate Analysis, 25: 286 - 298.

Bourguignon, F. (1999), "Comment to 'Multidimensioned Approaches to Welfare Analysis' by Maasoumi, E." in Handbook of income inequality measurement., ed. J. Silber, Boston, Dordrecht and London: Kluwer Academic, pp. 477-484.

Bourguignon, F., and S. R. Chakravarty. (2003), "The measurement of multidimensional poverty", Journal of Economic Inequality, 1 (1): 25-49.

Brandolini, A., and G. D'Alessio. (2001), "Measuring Well-Being in the Functioning Space" presented at the Conference on Justice and Poverty: Examining Sen's Capability Approach, 1998, Cambridge: unpublished.

Dardanoni, V. (1996), "On Multidimensional Inequality Measurement" in Research on Economic Inequality: Income Distribution, Social Welfare, Inequality and Poverty (Vol. 6), eds. C. Dagum and A. Lemmi, JAI Press Inc., pp. 201-205.

Frenkel, R. (2002), "Argentina: A Decade of the Convertibility Regime", Challenge, 45 (4): 41-59. 
Frenkel, R., and M. Gonzalez Rozada. (2000), "Tendencias de la distribución del ingreso en los años noventa", Serie de Documentos de Economía, Universidad de Palermo-CEDES (16).

Gajdos, T., and J. A. Weymark. (2005), "Multidimensional Generalized Gini Indices", Economic Theory, 26 (3): 471-496.

Gasparini, L. C. (2002), "Microeconometric Decompositions of Aggregate Variables: An Application to Labour Informality in Argentina", Applied Economics, 34 (18): 2257-2266.

Gonzalez Rozada, M., and A. Menendez. (2002), "Why have poverty and income inequality increased so much? Argentina 1991-2002", Documento de Trabajo, Universidad Torcuato Di Tella, Buenos Aires.

Heymann, D. (2000), "Políticas de reformas y comportamiento macroeconómico" in La Argentina de los noventa: desepeño económico en un contexto de reformas (Vol. 1), eds. D. Heymann and B. Kosacoff, Buenos Aires: Eudeba/CEPAL.

Hirschberg, J.-G., E. Maasoumi, and D.-J. Slottje. (2001), "Clusters of Attributes and Well-Being in the USA", Journal of Applied Econometrics, 16 (3): 445-460.

Hirschberg, J. G., E. Maasoumi, and D. J. Slottje. (1991), "Cluster Analysis for Measuring Welfare and Quality of Life across Countries", Journal of Econometrics, 50 (1-2): 131-150.

Kolm, S.-C. (1969), "The optimal production of social justice" in Public Economics, eds. J. Margolis and H. Guitton, London: Macmillan, pp. 145-200.

Kolm, S.-C. (1977), "Multidimensional Egalitarianism", The Quarterly Journal of Economics, 91 (1): 1-13.

Koshevoy, G., and K. Mosler. (1997), "Multivariate Gini Indices", Journal of Multivariate Analysis, 60: 252-276.

List, C. (1999), "Multidimensional inequality measurement: A proposal", Working Paper in Economics, Nuffield College, Oxford.

Lugo, M. A. (2004), "On Multivariate Distributions of Well-Being: The case of the Argentine Provinces in the 1990s" presented at the General Conference of The International Association for Research in Income and Wealth, August 22 - 28, Cork, Ireland.

Maasoumi, E. (1986), "The Measurement and Decomposition of Multi-dimensional Inequality", Econometrica, 54 (4): 991-997.

Maasoumi, E. (1993), "A Compendium to Information Theory in Economics and Econometrics", Econometric Reviews, 12 (2): 137-181. 
Maasoumi, E. (1999), "Multidimensioned Approaches to Welfare Analysis" in Handbook of income inequality measurement, ed. -. J. Silber, Boston: Kluwer Academic, pp. 437-477.

Maasoumi, E., and G. Nickelsburg. (1988), "Multivariate Measures of Well-Being and an Analysis of Inequality in the Michigan Data", Journal of Business and Economic Statistics, 6 (3): 326-334.

Marshall, A. W., and I. Olkin (1979), Inequalities : theory of majorization and its applications, Mathematics in science and engineering ; v. 143, New York: Academic Press.

Savaglio, E. (2006), "Three approaches to the Analysis of Multidimensional Inequality" in Inequality and Economic Integration, eds. F. Farina and E. Savaglio, London: Routledge.

Sen, A. K., and J. E. Foster (1997), On economic inequality (Enl. / ed.), Oxford: Clarendon Press.

Sen, A. K., G. Hawthorn, J. Muellbauer, S. M. R. Kanbur, K. Hart, and B. Williams (1987), The standard of living, The Tanner lectures on human values ; 1985, Cambridge: Cambridge University Press.

Shorrocks, A. F. (1980), "The Class of Additively Decomposable Inequality Measures", Econometrica, 48 (3): 613-625.

Tsui, K. Y. (1995), "Multidimensional Generalizations of the Relative and Absolute Inequality Indices: The Atkinson-Kolm-Sen Approach", Journal of Economic Theory, 67 (1): 251-265.

Tsui, K. Y. (1999), "Multidimensional Inequality and Multidimensional Generalized Entropy Measures: An Axiomatic Derivation", Social Choice and Welfare, 16 (1): 145-157.

UNDP (1995), Human Development Report, New York and Oxford: United Nations Development Programme.

Weymark, J. A. (2006), "The normative approach to the Measurement of Multidimensional Inequality" in Inequality and Economic Integration (July 2003 (revised January 2004) ed.), eds. F. Farina and E. Savaglio, London: Routledge.

Zamagni, S. (1987), Microeconomic theory : an introduction, Oxford: Basil Blackwell. 